\title{
Threat but not arousal narrows attention: evidence from pupil dilation and saccade control
}

\section{Henk van Steenbergen*, Guido P. H. Band and Bernhard Hommel}

Leiden Institute for Brain and Cognition and Leiden University Institute of Psychology, Leiden University, Leiden, Netherlands

Edited by:

Wim Notebaert, Ghent University, Belgium

\section{Reviewed by:}

Birgit Stürmer, Humboldt - Universität zu Berlin, Germany

Esther Aarts, University of California at Berkeley, USA

\section{*Correspondence:}

Henk van Steenbergen, Leiden Institute for Brain and Cognition and Leiden University Institute of Psychology, Leiden University, Wassenaarseweg 52, 2333 AK Leiden, Netherlands.

e-mail: hvansteenbergen@fsw. leidenuniv.nl

\begin{abstract}
It has been shown that negative affect causes attentional narrowing. According to Easterbrook's (1959) influential hypothesis this effect is driven by the withdrawal motivation inherent to negative emotions and might be related to increases in arousal. We investigated whether valence-unspecific increases in physiological arousal, as measured by pupil dilation, could account for attentional narrowing effects in a cognitive control task. Following the presentation of a negative, positive, or neutral picture, participants performed a saccade task with a pro-saccade versus an anti-saccade instruction. The reaction time difference between pro- and anti-saccades was used to index attentional selectivity, and while pupil diameter was used as an index of physiological arousal. Pupil dilation was observed for both negative and positive pictures, which indicates increased physiological arousal. However, increased attentional selectivity was only observed following negative pictures. Our data show that motivational intensity effects on attentional narrowing can occur independently of physiological arousal effects.
\end{abstract}

Keywords: arousal, focused attention, valence, pupil dilation, anti-saccade task

\section{INTRODUCTION}

In order to cope with threatening events, organisms often recruit extra resources. Regarding cognitive resources, there is evidence that affectively negative stimuli immediately prioritize the perceptual processing (Öhman et al., 2001) and recall (Christianson, 1992) of related information at the cost of other processes (Bocanegra and Zeelenberg, 2009; Pessoa, 2009), and it has been argued that these effects are mediated by the organism's current state of arousal (Schimmack, 2005). According to Easterbrook's (1959) influential hypothesis, increased arousal may lead to the narrowing and focusing of attention, thus facilitating appropriate subsequent responding and coping behavior.

Although threatening events and stress have been demonstrated to narrow attention (Cohen, 1980; Chajut and Algom, 2003; Gable and Harmon-Jones, 2010a; for a discussion of opposite effects in trait anxiety, see Pacheco-Unguetti et al., 2010), it is not clear whether these observations are due to non-specific arousal or the activation of affect-specific emotional/motivational systems (Bradley, 2000). Even though Easterbrook's original hypothesis relates to unpleasant situations only, several authors have suggested that any increase of arousal - e.g., whether induced by caffeine ingestion or impulsivity traits - may modulate attentional selectivity (e.g., Anderson, 1990). Along similar lines, increasing motivational intensity has been reported to increase focused attention irrespective of the motivational system (approach versus avoidance) involved (Gable and Harmon-Jones, 2008, 2010a,b; Harmon-Jones and Gable, 2009). However, whether arousal can be conceived of as a unitary construct has been questioned (Lacey, 1967; Neiss, 1988, 1990) and it is not entirely clear how motivational intensity and arousal are conceptually related (cf. Gable and Harmon-Jones, 2010b). Accordingly, it remains to be shown whether the emotional modulation of the selective attention reflects non-specific arousal that can vary orthogonally to the valence of the present affective state or whether it is specific to negative, potentially threatening events.

To index a possible narrowing of the attentional focus we used a visual anti-saccade task (for a review, see Hutton and Ettinger, 2006). This task is typically used as a measure of cognitive control, which is closely related to attentional selectivity. That is, the amount of information that is entering the focus of attention may be limited by cognitive control exerted at a perceptual or more central processing level (Desimone and Duncan, 1995; Miller and Cohen, 2001; Pessoa et al., 2003). Moreover, given that the neural mechanisms underlying the anti-saccade task are well known and can easily be studied in monkeys as well (cf. Munoz and Everling, 2004), we considered this task to be particularly well suited for studying the neuro-cognitive effects of emotion on attentional selectivity. As a first step, our study aimed at disentangling the relative contributions of arousal and affective valence on attentional control using physiological and behavioral measures of arousal and focused attention, respectively.

In the anti-saccade task, participants are presented with a peripheral, emotionally neutral target stimulus that appears with an abrupt onset on the left or right of the central fixation. Depending on the instruction, they are to move their eyes either to this target (pro-saccade condition) or to the opposite side of the display (anti-saccade condition). The common finding is that saccades are initiated more slowly and less reliably in the anti-saccade than in the pro-saccade condition. This cost is commonly attributed to the automatic tendency to look at novel events, which requires active inhibition in the anti-saccade condition (Olk and Kingstone, 2003). Because improved attentional control decreases automatic 
capture by the target stimulus (Yantis and Jonides, 1990), reflexive saccades toward the stimulus become suppressed. Thus, we expected that manipulations improving focused attention reduce the size of the latency costs, with anti-saccades becoming faster and pro-saccades becoming slower (cf., Kristjansson, 2007).

Affective states were induced prior to each saccade-task trial using positive, negative, or neutral pictures from the International Affective Pictures System (IAPS). Valence and arousal ratings of these pictures show a quadratic relationship, such that positive and negative stimuli are typically highly arousing and neutral stimuli low arousing (Lang et al., 2008). To ascertain that the pictures induced a physiological response we used pupillometry. Recent work by Bradley et al. (2008) has validated this approach. In that study, both negative and positive IAPS pictures were shown to produce pupil dilation, a response reflecting emotional arousal which is associated with increased sympathetic nervous activation. By means of this setup we were able to contrast two competing hypotheses. If more attentional selectivity in affectively laden circumstances would be driven by non-specific arousal, the difference in saccadic reaction time (RT) between anti- and pro-saccades should be reduced following negative as well as positive arousing pictures as compared to the non-arousing neutral pictures. Alternatively, if more attentional selectivity is specific to threatening situations, this latency cost should be reduced following negative stimuli but comparable for positive and neutral stimuli.

\section{MATERIALS AND METHODS PARTICIPANTS}

Eleven students from Leiden University (18-22 years old; two males; one left-handed) participated for either payment (5 Euros) or course credits.

\section{MATERIALS}

Thirty-two highly arousing negative, 32 neutral, and 32 highly arousing positive pictures were selected from the IAPS set (Lang et al., 2008 ${ }^{1}$ ). The stimulus set was almost identical to the one used by Bradley et al. (2008). Like that study, negative and positive stimuli could be differentiated on the basis of valence IAPS ratings, whereas they were matched for arousal IAPS ratings (Lang et al., 2008, see Table 1). Neutral pictures had low arousal ratings and intermediate valence ratings. In order to avoid light reflex confounds we used gray-scaled pictures (cf. Bradley et al., 2008); brightness and contrast were adjusted to ensure identical mean luminosity values for all pictures.

\section{TASK}

Each trial started as soon as participants had successfully looked at the central fixation cross for at least $1 \mathrm{~s}$. Then an IAPS stimulus

\footnotetext{
${ }^{1}$ The library numbers for the IAPS stimuli used in the present study are: Negative: 2120, 2205, 2520, 2590, 2691, 2730, 2750, 2800, 3015, 3030, 3053, 3100, 3170, 3180, $3181,3400,3500,3530,3550,6210,6211,6212,6821,6834,6838,9041,9250,9300$, 9341, 9405, 9800, 9921. Neutral: 2020, 2190, 2200, 2210, 2214, 2215, 2220, 2221, 2235, 2240, 2270, 2272, 2278, 2383, 2393, 2410, 2441, 2491, 2493, 2514, 2579, 2620 , 2749, 2752, 2810, 2850, 2870, 2890, 3210, 5455, 7550, 9210. Positive: 2208, 2250, 2260, 2501, 2560, 2650, 4611, 4617, 4640, 4650, 4653, 4658, 4659, 4689, 5621, 8041, $8080,8090,8116,8120,8161,8180,8200,8280,8300,8320,8330,8370,8380,8400$, 8420,8465 .
}

Table 1 | Emotion and performance measures as a function of picture content (table shows means and SE between brackets).

\begin{tabular}{|c|c|c|c|c|c|c|}
\hline & \multicolumn{6}{|c|}{ Picture content } \\
\hline & \multicolumn{2}{|c|}{ Negative } & \multicolumn{2}{|c|}{ Neutral } & \multicolumn{2}{|c|}{ Positive } \\
\hline \multicolumn{7}{|l|}{ SELF REPORT } \\
\hline Valence rating & 2.4 & $(0.11)$ & 5.0 & $(0.11)$ & 7.0 & $(0.11)$ \\
\hline Arousal rating & 5.9 & $(0.16)$ & 3.6 & $(0.16)$ & 5.5 & $(0.16)$ \\
\hline \multicolumn{7}{|l|}{ PHYSIOLOGY } \\
\hline Pupil diameter (mm) & 4.25 & $(0.040)$ & 4.19 & (0.026) & 4.23 & (0.033) \\
\hline \multicolumn{7}{|l|}{ BEHAVIOR } \\
\hline Pro-saccadic RT (ms) & 196 & (8.3) & 185 & (7.5) & 189 & (7.4) \\
\hline Anti-saccadic RT (ms) & 257 & $(12.7)$ & 260 & (10.8) & 265 & (9.8) \\
\hline
\end{tabular}

appeared for $500 \mathrm{~ms}$, which was replaced by the fixation cross for a jittered interval ranging from 1500 to $2500 \mathrm{~ms}$. Following a 200-ms blank gap (cf. Everling and Fischer, 1998), the target stimulus (also a cross) appeared for $500 \mathrm{~ms} 8^{\circ}$ to the left or right to the screen center. Then the central fixation cross appeared for another interval (ranging from 1000 to $2500 \mathrm{~ms}$ ) before the next trial started. At the beginning of each block an 8 -s verbal cue (approximately $5.7^{\circ} \times 1.4^{\circ}$; width $\times$ height) indicated whether a pro- (target position) or an anti-saccade (mirror position of the target) was to be made to the next target stimulus. The picture $\left(16^{\circ} \times 12^{\circ}\right)$ and the black fixation cross $\left(0.8^{\circ} \times 0.8^{\circ}\right)$ were shown on a gray background with luminosity equal to the mean of the pictures. In rare cases $(0.2 \%$ of the time), eye tracker recording problems delayed the trial presentation (inter-trial intervals $>9 \mathrm{~s}$ ). Because the interruption of the ongoing presentation sequence by either delayed presentation or by the onset of a new block is likely to compromise the experimental manipulation of the arousal and valence, the first five trials after such events were excluded.

\section{PROCEDURE}

Participants were instructed to attend to the emotional pictures and to make pro- and anti-saccades to the target as fast and accurate as possible. They were also asked to avoid eye blinks during picture and target presentation. After informed consent and eye tracker calibration, subjects practiced with six pro-saccade and six anti-saccade trials preceded by neutral IAPS pictures and followed by accuracy feedback for $1 \mathrm{~s}$. Calibration and/or practice were repeated in case of eye tracking problems or when the subject did not follow the instructions. The task consisted of six alternating pro-saccade and anti-saccade blocks (counterbalanced order), with two self-paced breaks in between. Each block consisted of 48 trials, and every chosen IAPS picture appeared three times in randomly chosen trials. Participants were debriefed after the experiment.

\section{DATA ACQUISITION AND ANALYSIS}

Saccadic behavior and pupil diameter were recorded at $120 \mathrm{~Hz}$ using a Tobii T120 eye tracker, which was integrated into a 17 -inch TFT monitor. Participants were seated at a distance of approximately $60 \mathrm{~cm}$ from the monitor while their head was stabilized by using a chin rest. Artifacts and blinks as detected by the eye 
tracker were corrected by using a linear interpolation algorithm. A saccade was considered to begin as soon as the horizontal angle exceeded $2^{\circ}$ and speed passed a $30^{\circ} / \mathrm{s}$ threshold. For all analyses, we excluded the following trials: trials including and following recording-related delays (see above), trials following performance errors, trials with saccadic RT outliers ( $<80$ or $>500 \mathrm{~ms}$ ), and trials where no saccades could be detected. Repeated-measures ANOVAs with the factors picture content (negative, neutral, positive) and task (pro versus anti) were run on pupil dilation and saccadic behavior measures. Paired $t$-tests were used for post hoc tests.

\section{RESULTS}

\section{PUPIL DILATION}

Following Bradley et al. (2008), pupil dilation to the picture content was measured after the initial light reflex. Dilation was defined as the mean pupil diameter in a window from 2 to $2.5 \mathrm{~s}$ after picture onset, using a $200 \mathrm{~ms}$ pre-picture baseline. As Table 1 shows, both negative and positive pictures caused dilation in comparison to neutral pictures. Analyses revealed a main effect of picture content $[F(2,20)=4.74, p<0.05$, MSE $=0.005]$, independent of task $[F(2,20)=1.02$ n.s., MSE $=0.003]$. Replicating Bradley et al. (2008), planned $t$-tests confirmed that arousing pictures (pooling the positive and negative condition) increased pupil diameter $[t(10)=2.49, p<0.05]$. As in that study, there was also a trend for negative pictures to induce more dilation than positive pictures $[t(10)=1.822, p=0.09]$. Using neutral pictures as comparison, separate $t$-tests indicated a significant dilation for negative pictures $[t(10)=2.487, p=0.032]$ and a marginal significant dilation for positive pictures $\left[t(10)=1.822, p_{\text {one-sided }}=0.049\right]$.

\section{SACCADIC BEHAVIOR}

See Table 1 for details. As usually found, correct saccadic RTs were slower during anti blocks than during pro blocks $[F(1,10)=77.08, p<0.001, \mathrm{MSE}=1073.76]$. More importantly, this task effect interacted with picture content $[F(2,20=3.82$, $p<0.05, \mathrm{MSE}=112.48]$. Planned $t$-test showed that the latency cost (anti-RT minus pro-RT) was not reduced for arousing pictures (pooling the positive and negative condition) versus neutral pictures $[t(10)=1.50, p=0.163]$. Instead, the latency cost was reduced following negative pictures only $[t(10)=2.84, p<0.02]$ in comparison to neutral pictures, but not for positive pictures $[t(10)=0.21$, n.s. $]$. As Figure 1 illustrates, relative to the neutral baseline, negative pictures slowed down pro-saccadic RT [11 ms; $t(10)=3.34, p<0.01]$ but did not significantly speed up antisaccadic RTs $[3 \mathrm{~ms} ; t(10)=0.71$, n.s.], whereas positive pictures did not make any reliable difference ( 4 and $5 \mathrm{~ms}$, respectively, all n.s.).

Task also affected the error rates $[F(1,10)=17.90, p<0.01$, MSE $=0.025]$ : subjects committed $18 \%$ erroneous saccades in anti-saccade blocks but only $1.5 \%$ in pro-saccade blocks. This effect did not interact with picture content $[F(2,20)=0.97$, n.s., $M S E=0.006]$.

To further test whether arousal might mediate any of these negative emotion effects we re-ran the analyses of correct saccadic RTs with strong versus weak pupil dilation as an additional factor. For this purpose, we categorized the trials following emotional pictures by means of a median split of the corresponding dilation

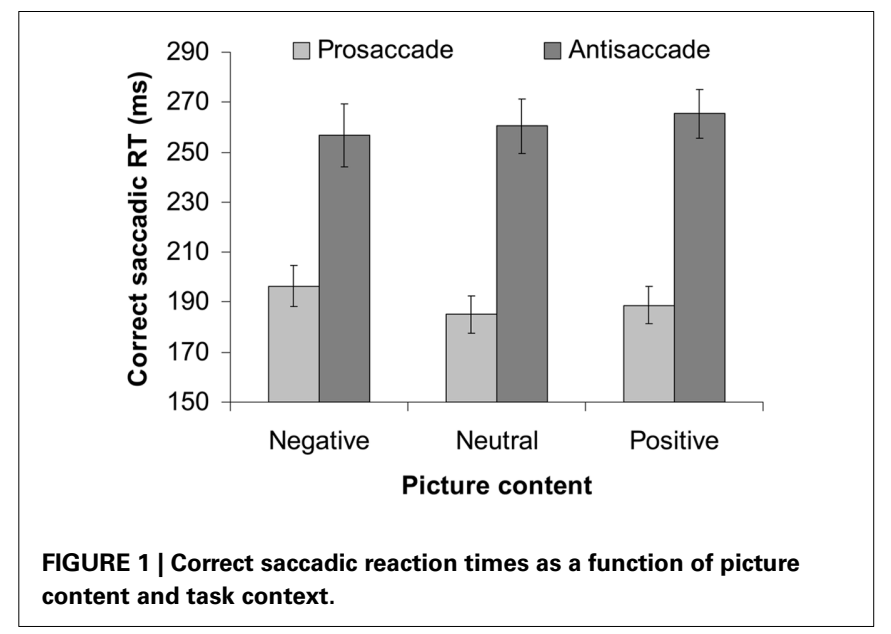

measures. However, even though we replicated the task effect and its interaction with picture content, the dilation factor was not involved in any main effect or interaction $(F s<1)$.

\section{DISCUSSION}

The aim of our study was to test whether attentional narrowing is due to general arousal or is selectively triggered by negative affective events. Although pupil dilation data confirmed that both negative and positive pictures increased the arousal level - a finding replicating Bradley et al. (2008) - attentional narrowing was observed following negative pictures only. This indicates that attentional narrowing is not caused by emotional arousal per se, at least as it can be measured by pupil dilation following the presentation of high-arousing pictures. In other words, increased emotional arousal may be a necessary condition, but it is not a sufficient condition for increased attentional selectivity. The same conclusion is suggested by the lack of impact of pupil dilation in the combined analysis. Hence, our observations do not provide any evidence for a role of arousal in driving attentional narrowing. Instead, the attentional focus seems to narrow whenever individuals are encountering events of negative affective valence.

How may negative affect regulate attentional narrowing? According to one account, dangerous situations may mobilize executive functions that protect against interference from disruption by irrelevant, distracting information (Norman and Shallice, 1986). Neuroimaging studies have suggested that these adjustments in cognitive control are implemented in the prefrontal cortex (Miller and Cohen, 2001), probably via signaling from the anterior cingulate cortex, a brain region involved in the detection of demanding and aversive situations (Botvinick et al., 2001; Shackman et al., 2011). Frontal cortex modulation, in turn, may modulate saccadic eye movements via the basal ganglia (Munoz and Everling, 2004). Thus, the reduced latency costs triggered by the negative pictures may originate from affect-driven modulation of cognitive control. This interpretation also fits earlier work that has used the anti-saccade task to assess inhibitory control (cf. Munoz and Everling, 2004). A similar explanation may also apply to earlier published studies such as effects on Stroop tasks usually attributed to attentional narrowing (e.g., Callaway, 1959; Agnew and Agnew, 1963; cf. Wachtel, 1967). 
However, it is important to emphasize that the reduced latency cost with negative pictures was driven by a slowing of RT during the pro-saccade block rather than a speeding of RT during the anti-saccade blocks. This indicates that processes other than improved control may also play a role in the affective modulation of behavior. For example, although it is likely that negative emotions increased control and attentional selectivity, which inhibits the visuo-motor grasp reflex resulting in delayed pro-saccadic RTs (Kristjansson, 2007), this effect may have become attenuated during the anti-saccade blocks. Because a state of high cognitive control is known to attenuate the effects of negative emotions (Ochsner and Gross, 2005), it might be that the effects of emotions on cognitive control were less pronounced in situations of higher task demands. Alternatively, it could be that the possible speeding of anti-saccades is masked by an overall slowing effect induced by the negative pictures. Indeed, several studies suggest that the processing of negative events may compete for perceptual and/or executive resources, which may slow down performance on a subsequent task (e.g., Gehring et al., 1993; Notebaert et al., 2009; Pessoa, 2009; Cohen et al., 2011). It remains an important aim for future studies to disentangle the role of these bidirectional interactions between emotions, perception, and executive function (cf. Vuilleumier et al., 2003).

\section{REFERENCES}

Agnew, N., and Agnew, M. (1963). Drive level effects on tasks of narrow and broad attention. Q. J. Exp. Psychol. $15,58-62$.

Anderson, K. J. (1990). Arousal and the inverted-U hypothesis - a critique of Neiss reconceptualizing arousal. Psychol. Bull. 107, 96-100.

Bocanegra, B. R., and Zeelenberg, R. (2009). Dissociating emotioninduced blindness and hypervision. Emotion 9, 865-873.

Botvinick, M. M., Braver, T. S., Barch, D. M., Carter, C. S., and Cohen, J. D. (2001). Conflict monitoring and cognitive control. Psychol. Rev. 108, 624-652.

Bradley, M. M. (2000). "Emotion and motivation," in Handbook of Psychophysiology, 2nd Edn, eds J. T. Cacioppo, L. G. Tassinary, and G. G. Berntson (Cambridge: Cambridge University Press), 602-642.

Bradley, M. M., Miccoli, L., Escrig, M. A., and Lang, P. J. (2008). The pupil as a measure of emotional arousal and autonomic activation. Psychophysiology 45, 602-607.

Callaway, E. (1959). The influence of amobarbital (amylobarbitone) and methamphetamine on the focus of attention. J. Ment. Sci. 105, 382-392.

Chajut, E., and Algom, D. (2003). Selective attention improves under stress: implications for theories of social cognition. J. Pers. Soc. Psychol. 85, 231-248.
Christianson, S. A. (1992). Emotional stress and eyewitness nemory - a critical review. Psychol. Bull. 112, 284-309.

Cohen, N., Henik, A., and Mor, N. (2011). Can emotion modulate attention? Evidence for reciprocal links in the attentional network test. Exp. Psychol. 58, 171-179.

Cohen, S. (1980). Aftereffects of stress on human performance and social behavior - a review of research and theory. Psychol. Bull. 88, 82-108.

Desimone, R., and Duncan, J. (1995). Neural mechanisms of selective visual attention. Annu. Rev. Neurosci. 18, 193-222.

Easterbrook, J. A. (1959). The effect of emotion on cue utilization and the organization of behavior. Psychol. Rev. 66, 183-201.

Everling, S., and Fischer, B. (1998). The antisaccade: a review of basic research and clinical studies. $\mathrm{Neu}$ ropsychologia 36, 885-899.

Friedman, R. S., and Forster, J. (2011). Limitations of the motivational intensity model of attentional tuning: reply to Harmon-Jones, Gable, and Price (2011). Psychol. Bull. 137, 513-516.

Gable, P., and Harmon-Jones, E. (2010a). The blues broaden, but the nasty narrows: attentional consequences of negative affects low and high in motivational intensity. Psychol. Sci. 21, 211-215.

Our study demonstrates for the first time that increased emotional arousal is not a sufficient condition to produce focused attention: pro-saccadic slowing presumably reflecting attentional narrowing was observed for negative affect, but not for positive affect. Consistent with this finding, and in contrast to a common misinterpretation, Easterbrook's (1959) original hypothesis attributed attentional narrowing not to general arousal but to a drive or motivation to withdraw. Given that positive emotions with approach-motivation have been shown to increase attentional focus (e.g., Gable and Harmon-Jones, 2008; for a review, see Gable and Harmon-Jones, 2010b), it is an important challenge for future research to determine which affective dimension, other than arousal accurately predicts attentional narrowing induced by positive emotions. In line with very recent discussions (cf. Friedman and Forster, 2011; Harmon-Jones et al., 2011), our results imply that it is now time to start research programs that search for emotional dimensions beyond valence and arousal that are responsible for tuning one's attentional scope.

\section{ACKNOWLEDGMENTS}

This research was supported by the Netherlands Organization for Scientific Research (NWO) to Guido P. H. Band.

Gable, P., and Harmon-Jones, E. (2010b). The motivational dimensional model of affect: implications for breadth of attention, memory, and cognitive categorisation. Cogn. Emot. 24, 322-337.

Gable, P. A., and Harmon-Jones, E. (2008). Approach-motivated positive affect reduces breadth of attention. Psychol. Sci. 19, 476-482.

Gehring, W. J., Goss, B., Coles, M. G. H., Meyer, D. E., and Donchin, E. (1993). A neural system for error detection and compensation. Psychol. Sci. 4, 385-390.

Harmon-Jones, E., and Gable, P. A. (2009). Neural activity underlying the effect of approachmotivated positive affect on narrowed attention. Psychol. Sci. 20, 406-409.

Harmon-Jones, E., Gable, P. A., and Price, T. F. (2011). Toward an understanding of the influence of affective states on attentional tuning: comment on Friedman and Forster (2010). Psychol. Bull. 137, 508-512.

Hutton, S. B., and Ettinger, U. (2006). The antisaccade task as a research tool in psychopathology: a critical review. Psychophysiology 43, 302-313.

Kristjansson, A. (2007). Saccade landing point selection and the competition account of proand antisaccade generation: the involvement of visual attention a review. Scand. J. Psychol. 48, 97-113.

Lacey, J. I. (1967). "Somatic response patterning and stress: some revisions of activation theory," in Psychological Stress: Issues in Research, eds M. H. Appley and R. Trumbull (New York: Appleton-Century-Crofts), 14-38.

Lang, P. J., Bradley, M. M., and Cuthbert, B. N. (2008). International Affective Picture System (IAPS): Affective Ratings of Pictures and Instruction Manual. Technical Report A8. Gainesville, FL: University of Florida.

Miller, E. K., and Cohen, J. D. (2001). An integrative theory of prefrontal cortex function. Annu. Rev. Neurosci. 24, 167-202.

Munoz, D. P., and Everling, S. (2004). Look away: the anti-saccade task and the voluntary control of eye movement. Nat. Rev. Neurosci. 5, 218-228.

Neiss, R. (1988). Reconceptualizing arousal - psychobiological states in motor performance. Psychol. Bull. 103, 345-366.

Neiss, R. (1990). Ending arousals reign of error - a reply. Psychol. Bull. 107, 101-105.

Norman, D. A., and Shallice, T. (1986). "Attention to action: willed and automatic control of behavior," in Consciousness and Self-Regulation, eds R. J. Davidson, G. E. Schwartz, and D. Shapiro (New York: Plenum Press), 1-18. 
Notebaert, W., Houtman, F., Van Opstal, F., Gevers, W., Fias, W., and Verguts, T. (2009). Post-error slowing: an orienting account. Cognition 111, 275-279.

Ochsner, K. N., and Gross, J. J. (2005). The cognitive control of emotion. Trends Cogn. Sci. (Regul. Ed.) 9, 242-249.

Öhman, A., Flykt, A., and Esteves, F. (2001). Emotion drives attention: detecting the snake in the grass. J. Exp. Psychol. Gen. 130, 466-478.

Olk, B., and Kingstone, A. (2003). Why are antisaccades slower than prosaccades? A novel finding using a new paradigm. Neuroreport 14, 151-155.

Pacheco-Unguetti, A. P., Acosta, A., Callejas, A., and Lupianez, J. (2010). Attention and anxiety: different attentional functioning under state and trait anxiety. Psychol. Sci. 21, Vuilleumier, P., Armony, J., and Dolan, 298-304.

Pessoa, L. (2009). How do emotion and motivation direct executive control? Trends Cogn. Sci. (Regul. Ed.) 13, 160-166.

Pessoa, L., Kastner, S., and Ungerleider, L. G. (2003). Neuroimaging studies of attention: from modulation of sensory processing to top-down control. J. Neurosci. 23, 3990-3998.

Schimmack, U. (2005). Attentional interference effects of emotional pictures: threat, negativity, or arousal? Emotion 5, 55-66.

Shackman, A. J., Salomons, T. V., Slagter, H. A., Fox, A. S., Winter, J. J., and Davidson, R. J. (2011). The integration of negative affect, pain and cognitive control in the cingulate cortex. Nat. Rev. Neurosci. 12, 154-167.
R. (2003). Reciprocal links between emotion and attention," in Human Brain Function, ed. R. S. J. Frackowiak (San Diego: Academic Press), 419-444.

Wachtel, P. L. (1967). Conceptions of broad and narrow attention. Psychol. Bull. 68, 417-429.

Yantis, S., and Jonides, J. (1990). Abrupt visual onsets and selective attention - voluntary versus automatic allocation. J. Exp. Psychol. Hum. Percept. Perform. 16, 121-134.

Conflict of Interest Statement: The authors declare that the research was conducted in the absence of any commercial or financial relationships that could be construed as a potential conflict of interest.
Received: 27 June 2011; accepted: 05 October 2011; published online: 31 October 2011.

Citation: van Steenbergen $H$, Band GPH and Hommel B (2011) Threat but not arousal narrows attention: evidence from pupil dilation and saccade control. Front. Psychology 2:281. doi: 10.3389/fpsyg.2011.00281

This article was submitted to Frontiers in Cognition, a specialty of Frontiers in Psychology.

Copyright (C) 2011 van Steenbergen, Band and Hommel. This is an open-access article subject to a non-exclusive license between the authors and Frontiers Media $S A$, which permits use, distribution and reproduction in other forums, provided the original authors and source are credited and other Frontiers conditions are complied with. 